\title{
Study on Adaptability of Urban Agglomeration Freight transport Supply and Demand Structure Based on Entropy Theory
}

\author{
LIU Zhen-yu ${ }^{1, a}$, GAO Wei ${ }^{1, b}$, GAO Yue-yuan ${ }^{1, c}$, WANG Jing-jing ${ }^{1, d}$ \\ ${ }^{1}$ School of Transportation, Inner Mongolia University, Hohhot Inner Mongolia, 01007, China \\ a email:44855513@qq.com, b email:981749142@qq.com, cemail:3155228462@qq.com, d \\ email:1587301559@qq.com
}

\begin{abstract}
Keywords: urban agglomeration; freight transportation; co-ordination of supply and demand; information entropy theory; adaptability evaluation model

Abstract: To study the adaptability of freight transport supply and demand structure of urban agglomerations, the mechanism of demand and supply of freight transport in urban agglomeration was studied and the theory of freight transport structure of urban agglomeration was put forward and demonstrated. First, the main factors that affect the freight transport demand structure and supply structure of urban agglomeration was analyzed through information entropy theory. Second, the urban agglomeration freight transport demand structure and supply structure models was established based on information entropy. Then, through Comparing the freight transport demand structure entropy and supply structure entropy, adaptability evaluating model of freight transport supply and demand structure of urban agglomeration was proposed. Finally, taking H-B-E city agglomeration as an example of our empirical study, the method of adaptive evaluation and optimization model was used to analyze the freight transport supply and demand structure of the city agglomerations.
\end{abstract}

\section{Introduction}

With the development of urban agglomeration, the leading role of urban agglomeration transportation in the development of urban agglomeration is becoming increasingly prominent. As an important part of urban traffic, freight transportation attracts more and more attention. At present, the total effective demand is insufficient, the supply structure is irrational and the imbalance of supply and demand of traffic structure is prominent in urban agglomeration. Various modes of freight transportation cannot be coordinated in a reasonable proportion. The one-sided pursuit of maximization of self-interest makes the problem of adaptability of freight supply and demand structure prominent.

Based on the research on urban agglomeration freight supply and demand structure, there are few domestic and international research results. Literature[1-3] applies the theory of information entropy to the transportation industry, which studies the equilibrium problem of transportation supply and demand structure and the optimization scheme. Literature [4] studies the adaptive relationship between transportation and socio-economic development, and analyzes the equilibrium of supply and demand structure from two aspects of freight transportation and passenger transport. Literature [5-7]puts forward the forecast method of freight demandand analyzes the supply and demand equilibrium problem of freight transport. Literature [8] based on the PESTEL model and the implicit economic gravity model, analyze the traffic demand of the cities in Sichuan, and design the framework of the traffic network.

In conclusion, most of domestic and abroad research about freight transport supply and demand structure have been based on a single city and rarely through the city agglomeration perspective. By analyzing the factors affecting urban agglomeration freight transport demand and supply, the entropy models of urban agglomeration freight demand structure and supply structure was established based on entropy theory. Then, the equilibrium model of the two entropy is determined, and the non-equilibrium problem of the freight supply and demand structure of the urban agglomeration is analyzed intuitively.The adaptation conditions based on the equilibrium of urban freight supply and demand are obtained, which provides a theoretical basis for the balanced structure of urban agglomeration freight supply and demand structure. 


\section{Basic description of urban agglomeration freight supply and demand adaptability}

The research scope of urban agglomeration freight supply and demand includes three parts: internal transport supply and demand of a city inside urban agglomeration, intercity transport supply and demand between cities within urban agglomeration, intercity transport supply and demand within urban agglomeration with other cities outside urban agglomeration. In different stages of social and economic development, freight transport will form different demand structure, which requires all kinds of transport modes in a certain proportion relationship to adapt to the demand structure if the supply structure is relatively stable. If the transport demand for goods are to be met, freight supply and demand structure adaptation state is formed.

At present, the overall supply and demand imbalance of the freight market in China's urban agglomerations is prominent. The main manifestations are as follows: (1) the shortage of some traffic modes and the excess supply of some modes of transportation coexist. (2) eachtransportation mode emphasize their respective technical and economic advantages, seeking the maximum utility of their own investment, rather thanthe utility maximization of the entire transport industry. (3) The efficiency and utility of resources in different transportation modes vary greatly, and the overall efficiency are not high. Al of these will make the urban agglomeration supply and demand imbalanced. Therefore, the utility maximization of urban agglomeration transportation was studied considering the urban agglomeration as a whole, which can provide a theoretical basis for the urban agglomeration freight transport supply and demand disequilibrium problem.

\section{Entropy model of urban agglomeration freight supply and demand structure}

\section{The factors affecting urban agglomeration freight transport demand structure}

\section{(1) urban agglomeration industrial structure}

The change of industry structure is the root of the change of the transport structure. Due to the adjustment in the ratio of various industries, type, flow and direction of cargoes are all going to be different, and then the transport demand of city agglomeration will change affecting the adaptability of transport supply and demand structure.

(2) urban agglomeration production distribution

The layout of certain productivity distribution requires different transportation modes to complete transportationof all kinds of raw materials and products. With the change of the production distribution, some freight transport need to rely on some other sort of transport with their demand declares, or replaceby other modes of transport, so the original transport structure is broken.

(3) Other influencing factors

Domestic economic policy, energy structure and transportation technology will indirectly affect the structure changes in freight transport demand, but these factors are relatively stable in the short term.

\section{Establishment of urban agglomeration freight demand structure entropy model}

The freight demand structure of urban agglomerations can be regarded as a function with the freight demand structure influence factors as variables, thus the function expression of freight demand structure of urban agglomerations is obtained using Equation (1).

$$
D(\dot{d})=f(\dot{\prime}, \stackrel{\prime}{L}, \varepsilon)
$$

Where, $d$ represents traffic demand structure vector of four transportation modes; I represents urban agglomeration industrial structure vector; L represents urban agglomeration production distribution vector; $\varepsilon$ represents the other influencing factors of urban agglomeration freight demand structure.

(1) Entropy model of urban agglomeration industrial structure

According to the definition of information entropy, the entropy of industrial structure is calculated by weighting the weights of different industrial structure to reflect the industrial change 
of urban agglomeration in different stages of economic development, and the entropy of industrial structure in $t$ period is obtained as Equation (2).

$$
H_{i}(t)=-\sum_{i=1}^{3} P_{j t} \ln P_{j t}
$$

Among them, the proportion of industrial $j$ in $t$ period is $P_{j t}$.

$$
\begin{gathered}
P_{j t}=I_{j t} / \sum_{j=1}^{3} I_{j t} \\
I_{j t}=\sum_{i=1}^{n} I_{i j t}
\end{gathered}
$$

Where, $I_{j t}$ represents output value of industry $j$ in period $t$ in urban agglomeration; $I_{i j t}$ represents output value of industry $j$ in period $t$ in city $i ; P_{j t}$ represents the weight of industry $j$ at period $t(\mathrm{j}=1,2,3$,representing the first industry, the second industry, the third (2) industry).

model

Urban agglomeration production distribution structure

The production distribution of four parts: the layout of urban agglomerations, the layout of metallurgical industry, the layout of power industry and the layout of petroleum refining industry is analyzed. The changes in the productivity structure of the urban agglomerations during the period $t_{\text {can }}$ be mapped to the four modes of transportation as Equation (5).

$$
\begin{aligned}
& \check{L}_{(t-1)}=\left[L_{1(t-1)}, L_{2(t-1)}, L_{3(t-1)}, L_{4(t-1)}\right] \rightarrow \bar{d}_{(t-1)}=\left[d_{1(t-1)}, d_{2(t-1)}, d_{3(t-1)}, d_{4(t-1)}\right] \\
& \check{L}_{(t)}^{\mathbf{v}}=\left[L_{1(t)}, L_{2(t)}, L_{3(t)}, L_{4(t)}\right] \rightarrow \check{d}_{(t)}=\left[d_{1(t-1)} \pm e_{1}, d_{2(t-1)} \pm e_{2}, d_{3(t-1)} \pm e_{3}, d_{4(t-1)} \pm e_{4}\right] \\
& e_{i t}=\sum_{i=1}^{n} e_{g i t}
\end{aligned}
$$

Urban agglomeration productivity distribution structure entropy in period $t$ is $H_{L(t)}$.

$$
\begin{gathered}
H_{L(t)}=-\sum_{x=1}^{4} P_{t}^{\prime} \operatorname{In}^{P_{t}^{\prime}} \\
P_{t}^{\prime}=\frac{\left[d_{i(t-1)} \pm e_{i}\right]}{\sum_{x=1}^{4}\left[d_{i(t-1)} \pm e_{i}\right]}
\end{gathered}
$$

Where, $\check{L}_{(t-1)}$ represents the urban agglomeration production distribution structure vector at period $t-1 ; d_{(t-1)}$ represents the structure vector of the demand of various traffic modes corresponding to the production distribution of urban agglomeration at period $t-1$; $d_{i(t-1)}$ represents the traffic volume corresponding to the traffic structure of the urban agglomeration in period $t-1 ; d_{g(t-1)}$ represents the traffic volume corresponding to the traffic structure of a city in period $t-1 ; \boldsymbol{e}_{i t}$ represents the change of the demand degree of the corresponding traffic mode caused by the change of industry distribution of the urban agglomeration in period $t-1$; $\boldsymbol{e}_{\text {git }}$ represents the change of the demand degree of the corresponding traffic mode caused by the change of industry distribution of a city in period $t-1$ (traffic modes include roads, railways, airlines, water transportation).

According to the above calculation of the influence factors of urban agglomeration freight demand entropy, we can get the structure entropy model of freight transport demand structure of urban agglomeration as Equation (8).

$$
H_{d}(t, d)=a H_{I(t)}+b H_{L(t)}+\varepsilon_{(t)}
$$

Where, $a 、 b$ are undetermined coefficients. 


\section{Establishment of entropy model for urban agglomeration freight supply structure Urban Agglomeration}

\section{Analysis of main influencing factors of urban agglomeration freight supply structure}

(1) The influence of fixed assets structure on freight supply structure

The fixed assets constitute the material basis of the production capacity of each mode of freight transport, which reflects the size of the production capacity. Under the established structure, the fixed assets structure mainly depends on the investment structure, and can be expressed by the structure vector of the assets investment.

$$
A(t)=\left[A_{1}(t), A_{2}(t), A_{3}(t), A_{4}(t)\right]
$$

Combined with the definition of entropy, the structure of fixed assets can be used to represent the investment of different modes of transportation.

$$
\begin{gathered}
H_{A i}=-\sum_{i}^{4} P_{j} \operatorname{In} P_{j} \\
P_{j}=\frac{A_{i j}}{\sum_{i=1}^{4} A_{i j}}
\end{gathered}
$$

Where, $A(t)$ represents fixed assets structure; $A_{i j}$ represents quota allocation of fixed assets investment in various modes of transportation(including roads, railways, aviation and water transport mode); $H_{A i}$ represents fixed investment structure entropy of traffic system; $P_{j}$ represents the fixed assets investment weight of goods transportation mode $j$.

(2) The influence of technical and economic structure on freight supply structure

Due to the technical characteristics of all kinds of freight transportation (such as transport speed, transport capacity, etc.), the economic characteristics (such as transportation, capital occupation etc.), each transportation modes have their own comparative advantages and scope of use, which affects the transport structure. Freight technical and economic structure vector is as follows.

$$
T(t)=f[V(t), P(t), C(t)]
$$

Where, $T(t)$ represents freight technical and economic structure vector; $V(t)$ represents the transport velocity vector of various freight modes; $P(t)$ represents the unit carrying capacity of various modes of freight transport; $C(t)$ represents the unit transportation cost of various modes of freight transportation.

\section{Entropy model of urban agglomeration freight supply structure}

The indexes reflecting freight supply structure are as follows:

$$
X(t)=\left[\begin{array}{l}
x_{11}(t) x_{12}(t) x_{13}(t) x_{14}(t) \\
x_{21}(t) x_{22}(t) x_{23}(t) x_{24}(t) \\
x_{31}(t) x_{32}(t) x_{33}(t) x_{34}(t) \\
x_{41}(t) x_{42}(t) x_{43}(t) x_{44}(t)
\end{array}\right]
$$

Where, $X(t)$ represents the matrix of freight supply structure index value in period $t$; $x_{i j}(t)$ represents index value $j$ of freight mode $i$ in period $t ; i=1,2,34$ respectively represents 4 modes of freight transport, including fixed assets investment, freight transport speed, unit carrying capacity and unit transportation cost.

Because each index has different dimensions, it is necessary to standardize the value of each index in the matrix, and the standardized method is as follows.

$$
\begin{array}{r}
p_{i j}(t)={ }^{x_{i j}}(t) / \sum_{i=1}^{4} x_{i j}(t) \\
\sum_{i=1}^{4} p_{i j}(t)=1
\end{array}
$$


The normalized matrix is obtained.

$$
P(t)=\left[\begin{array}{ll}
p_{11}(t) \mathrm{L} & p_{14}(t) \\
\mathrm{M} & \\
p_{41}(t) \mathrm{L} & p_{44}(t)
\end{array}\right]
$$

According to the meaning of information entropy, the entropy value of the index is:

$$
H\left(t, p_{j}\right)=-\sum_{i=1}^{4} p_{i j}(t) \ln p_{i j}(t)
$$

Define $0 \ln 0=0$, when $p_{1 j}(t)=p_{2 j}(t)=\mathrm{L} p_{4 j}(t)=1 / 5$, the amount of information contained in the index is consistent with the study area. The output entropy reaches its maximum value $H\left(t, p_{j}\right) / \ln 4 . H_{j}(t)=H\left(t, p_{j}\right)=\ln 4,0 \leq H_{j}(t) \leq 1 . H_{j}(t)$ represents the equilibrium degree of freight supply structure based on the index of information entropy function. $j$ represents information utility value of index, which depended on the difference between the index equilibrium $H_{j}(t)$ and 1.

$$
G_{j}(t)=1-H_{j}(t)
$$

Calculate the entropy weight of index $j$

$$
w_{H}^{j}=G_{j} / \sum_{i=1}^{4} G_{i}
$$

The index vector based on entropy weight is obtained.

$$
W_{H}=\left(w_{H}^{1}, \mathrm{~L} w_{H}^{4}\right)
$$

The freight supply vector can be obtained by index normalization matrix $P(t)$ and entropy weight vector $W_{H}(t)$ based on the Fuzzy mathematical theory. By using the fuzzy composition operator to calculate the vector, the contribution degree of various freight modes to freight supply structure can be calculated.

The actual freight volume of four kinds of freight transportation is taken as the supply of urban agglomeration freight, and $x_{1}, x_{2}, x_{3}$, is used to express the traffic weight coefficient (internal transport supply and demand of a city inside urban agglomeration, intercity transport supply and demand between cities within urban agglomeration, intercity transport supply and demand within urban agglomeration with other cities outside urban agglomeration). And the structure vector of the urban agglomeration freight supply is obtained.

$$
\begin{gathered}
S=\left(S_{1}, S_{2}, S_{3}, S_{4}\right) \\
S_{i}=X_{1} \sum_{i=j=1}^{m} s(i, j)+X_{2} \sum_{i=1}^{m} \sum_{j=1}^{n} s(i, j)+X_{3} \sum_{i=1}^{m} \sum_{j=m}^{n} s(i, j)
\end{gathered}
$$

Where, $S_{i}$ represents the freight volume of urban agglomeration (roads, railways, aviation and water transport mode); $S_{i}(i, j)$ represents traffic volume of mode $i$ between city $s i$ and city $s j$ in urban agglomeration.

The entropy model of freight supply structure of urban agglomeration is obtained.

$$
H_{s}(t, s)=-\sum_{i=1}^{4} \frac{T_{i}}{\ln T_{i}}
$$

The proportion of freight mode $i$ is $T_{i}=$

$$
s_{i} / \sum_{i=1}^{4} s_{i}
$$

\section{Adaptability determination of urban agglomeration freight supply and demand structure}

In order to determine the degree of adaptability between freight supply structure and freight demand 
structure, it is necessary to compare freight supply structure and freight demand structure. Because the freight demand structure and freight supply structure are all functions of time, the adaptability evaluation model of freight supply and demand structure of period $t$ is established.

$$
G(t)=\left|1-\frac{H_{s}(t, s)}{H_{d}(t, d)}\right|
$$

The ratio of freight supply structure entropy and freight demand structure entropy is closer to 1, the approximation degree is greater, the adaptability of freight supply and demand structure is stronger. In this way, the minimum structure 0 is obtained when $H_{d}(t, d)=H_{s}(t, s)$, and freight supply structure is the most adaptable to freight demand structure.

According to the literature [2], the adaptability of urban agglomeration freight supply and demand structure can be divided into the following grades.

Table1 Adaptability degree of urban agglomeration freight supply and demand structure

\begin{tabular}{|c|c|c|}
\hline types & Adaptive type & $\mathrm{G}(\mathrm{t})$ \\
\hline \multirow{3}{*}{ adaptability } & High quality adaptation & $0.9<\mathrm{G}(\mathrm{t}) \leq 1$ \\
\cline { 2 - 3 } & Good adaptation & $0.8<\mathrm{G}(\mathrm{t}) \leq 0.9$ \\
\cline { 2 - 3 } & Basic adaptation & $0.7<\mathrm{G}(\mathrm{t}) \leq 0.8$ \\
\hline \multirow{3}{*}{ inadaptability } & Basic inadaptability & $0.6<\mathrm{G}(\mathrm{t}) \leq 0.7$ \\
\cline { 2 - 3 } & inadaptability & $0.4<\mathrm{G}(\mathrm{t}) \leq 0.6$ \\
\cline { 2 - 3 } & Terribly inadaptability & $0<\mathrm{G}(\mathrm{t}) \leq 0.4$ \\
\hline
\end{tabular}

\section{case analysis}

In order to confirm the above method, H-B-O urban agglomeration is taken as an example to verify urban agglomeration freight supply and demand structure adaptive. The urban agglomeration includes Hohhot, Baotou, Ordos, Ulanqab and Wuhai. In the process of calculation, considering the convenience of data acquisition and calculation, the influence of urban agglomeration industrial structure on freight demand structure of urban agglomeration is considered only.

(1) Calculation of urban agglomeration freight demand structure entropy

Table2 The three major industries of cities in H-B-O urban agglomeration in 2015

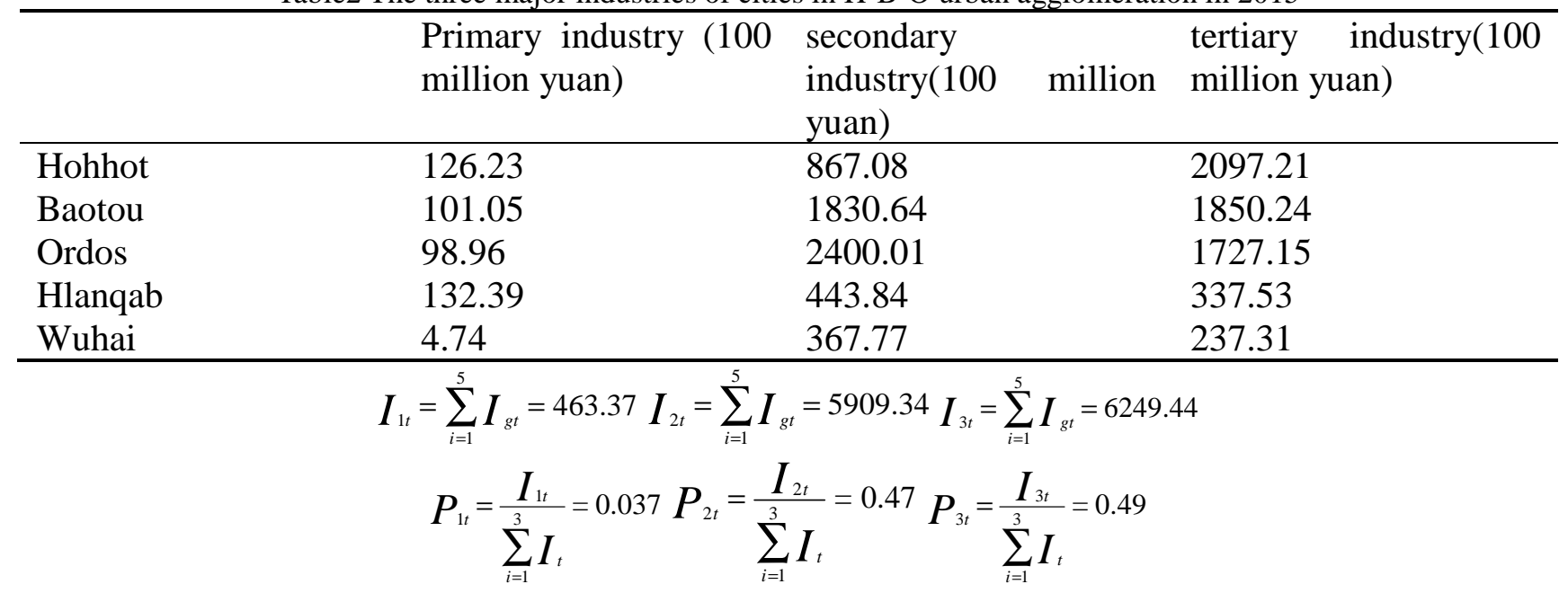

The urban agglomeration industrial structure entropy is obtained.

$$
H_{\mathrm{t}}=-\sum_{\mathrm{j}=1}^{3} \mathrm{P}_{j} \ln \mathrm{P}_{j}=0.82
$$


(2) Urban agglomeration freight supply structure entropy

Table3 Freight turnover of cities in H-B-O urban agglomeration in 2015

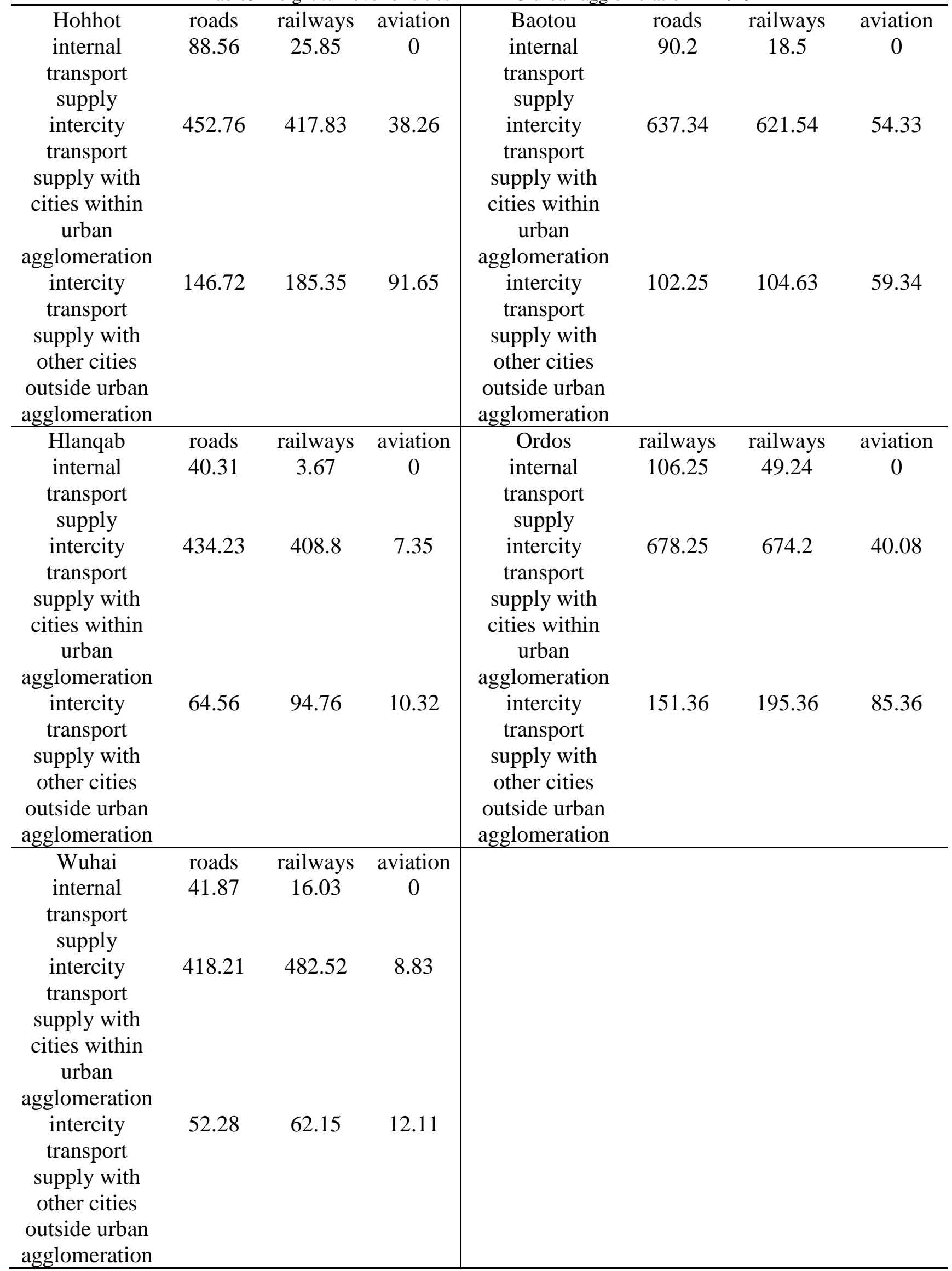




$$
\begin{gathered}
S_{1}=\sum_{i=1}^{5} S_{1 j}=3503.15 S_{2}=\sum_{i=1}^{5} S_{2 j}=3360.43 S_{3}=\sum_{i=1}^{5} S_{3 j}=407.63 \\
T_{1}=s_{1} / \sum_{i=1}^{3} s_{i}=0.48 T_{2}=s_{2} / \sum_{i=1}^{3} s_{i}=0.46 T_{3}=s_{3} / \sum_{i=1}^{3} s_{i}=0.06
\end{gathered}
$$

The urban agglomeration freight supply structure entropy is obtained.

$$
H_{s}(t)=-\sum_{i=1}^{3} T_{i} \ln T_{i}=0.88
$$

According to the freight demand structure entropy and freight supply structure entropy of H-B-O urban agglomeration, the H-B-O urban agglomeration non-equilibrium degree of traffic supply and demand $o$ is obtained.

$$
G(t)=\left|1-\frac{H_{(t)}}{H_{s}(t)}\right|=0.07
$$

The calculation result shows that the adaptability of freight supply and demand structure in H-B-O urban agglomeration is in terribly indaptability, so there is a big difference between the freight supply structure and the freight demand structure.

\section{Conclusion}

Freight transport demand structure and freight trans supply structure have a certain mutual correspondence. That is to say, the total freight supply will meet the total demand of freight, and the supply structure should adapt to the demand structure. If the entropy value of freight supply structure is consistent with the entropy of freight demand structure, the value of adaptability function $G(t)$ should tend to 1. Based on information entropy theory, the freight supply and demand structure of urban agglomeration is taken as the research object, andthe adaptability model of freight supply and demand structure is put forward, which can provide a basic method for optimizing the structure of freight transportation.

\section{Acknowledgements}

This work was financially supported by the Inner Mongolia Autonomous Region higher school science and technology research projects (Project No: NJZY17012) and theCollege Students' innovation and Entrepreneurship Project (Project No: 201718407).

\section{References}

[1] Tang Jianqiao, Wang Ciguang. Adaptability between supply structure and demand structure of regional freight based on information entropy theory $[\mathrm{J}]$. Journal of highway and transportation research and development, 2013,30(4):148-154.

[2] Yu shi.Evolution and dynamic analysis of regional transportation structure information entropy[J]. Journal of transportation systems engineering and information technology,2011.

[3] Wu Feng, Shi Qizhou. Entopy theory based study on relationship between transportation and industry systems $[\mathrm{J}]$. Journal of transportation systems engineering and information technology,2006,6(1):71-74.

[4] Yuan Jing. Study on the structure equilibrium of transportation supply and demand[D]. Chang'an university,2007.

[5] Sun Fenghua. Study on forecasting method of regional freight demand[D]. Chang'an university,2011.

[6] Li Hongjunan. Economic analysis of demand and supply of urban freight transportation.[D]. Chang'an university,2010.

[7] Feng Yu. Study on freight transportation market demand and supply mechanism[D]. Maritime Affairs University Of Dalian,2006.

[8] Liu Zhijie. A study on transportation system planning for urban agglomerations[D].Chang'an 
university,2011. 